\title{
The direct measurement using an imaging plate for coincidence of radiation center and laser position in external radiation therapy.
}

Short title: $Q A$ by IP for external radiation therapy

\author{
Toshiyuki Terunuma, Takeji Sakae, Akihiro Nohtomi and Yoshikazu Tsunashima \\ Proton Medical Research Center (PMRC), University of Tsukuba, Ten-nohdai 1-1-1, Tsukuba 305-8575, Japan
}

E-mail: terunuma@pmrc.tsukuba.ac.jp

\begin{abstract}
A new method of quality assurance has been studied to measure coincidence of the radiation center and a patient-setup laser position on a transverse plane to the beam at the isocenter. This measurement is achieved by using an imaging plate (IP). When a radiation is applied to an IP, the energy is stored as trapped electrons. The number of electrons is decreased by local laser exposure. As a result, the radiation field produced by external beam irradiation is recorded as "positive" information and the position of the patient-setup laser is recorded as "negative" one on an IP. The advantages of this method are the direct measurement, short time and high resolution. These are required for daily and monthly quality checks. We confirmed the advantage of this method by an experiment using proton beam.
\end{abstract}

\section{Introduction}

In external radiation therapy, the target is most commonly positioned in the desired location with help of patient-setup lasers, which indicate the position of the isocenter. Therefore, it is very important to verify and control coincidence of the radiation center and the laser position accurately. Many studies have been made on the quality assurance of this subject (Tsai et al. 1996, Arjomandy et al. 2000, Treuer et al. 2000, Welsh et al. 2002). In the previous works, some objects were located on the laser position to know the coincidence. These additional setup procedures produced a new measurement error. Falco et al. (1999) developed a technique for the direct setup alignment of radiosurgical circular field from an isocentric linac to treatment room laser cross-hairs. But the laser cross-hairs did not superimposed in the radiosurgical field. A method realizing direct measurement of the coincidence of the radiation center and the laser position has been required.

We have tested a direct measurement by using an imaging plate (IP). An IP has been developed as 
an image storing medium and is now widely used as detectors of two-dimensional images produced by ionizing radiation in medical, biological and physical fields. In recent years, it found that an IP is also applicable to a proton beam (Hayakawa et al. 1996, Nohtomi et al. 1999 and 2000). An IP has a flexible polyester base coated with highly dispersed barium fluorohalide phosphor crystal $\mathrm{BaFBr}: \mathrm{Eu}^{2+}$ (Iwabuchi et al. 1994). Figure 1 shows the energy level diagram and photo-stimulated luminescence (PSL) process in $\mathrm{BaFBr}: \mathrm{Eu}^{2+}$. When a radiation is applied to an IP, the energy is transferred to the phosphor crystals and is stored as trapped electrons. This means that electrons become trapped to 'F-centers' in the $\mathrm{BaFBr}$ matrix and holes become trapped to $\mathrm{Eu}^{2+}$ ions. The radiation image is temporarily stored in the IP. These F-centers have an absorption band at about $600 \mathrm{~nm}$. The IP is read out by an image reader which scans the IP surface with a focused $633 \mathrm{~nm}$ He-Ne laser beam. The He-Ne laser releases the trapped electrons and recombine with the holes trapped by $\mathrm{Eu}^{2+}$, and photons at about 400nm are released. This is known as PSL phenomenon. The emitted PSL light is collected by a photomultiplier tube, and the tube output is recorded as time series of digital signals using with an analog-to-digital converter.

We expected that our patient-setup laser $(635 \mathrm{~nm})$ also releases the trapped electrons locally and this local reducing process can be applicable to record the laser position.

\section{Materials and Methods}

The experiment was carried out at a treatment room in the Proton Medical Research Center (PMRC), University of Tsukuba. A proton beam with energy of $200 \mathrm{MeV}$ was produced by a synchrotron and it passed through a dual-ring double-scatterer system to obtain a laterally uniform spatial distribution. As shown by figure 2 , a brass collimator with $10 \times 10 \mathrm{~cm}^{2}$ aperture and thickness of $5 \mathrm{~cm}$ was mounted onto the gantry head. An IP with $20 \times 25 \mathrm{~cm}^{2}$ sensitive area (BAS III, Fuji film Co. Ltd.) was placed on the transverse plane to the beam at the isocenter. The IP was irradiated only 1 spill of proton beam and the two-dimensional dose distribution was recorded. After proton beam irradiation, the laser system LDTF163C06-2 (Takenaka Optonic Co. Ltd.) was inserted on the beam line in the gantry head. Then the IP was exposed to the cross-hairs laser with wavelength of $635 \mathrm{~nm}$ and $0.1 \mathrm{~mW}$ power for about 20 seconds. These operations were performed in a darkened ambience in order to avoid light exposure reducing PSL signals. The IP was not covered to enable us laser exposure. The stored image was read out by BAS2000-II (Fuji film Co. Ltd.) with $100 \mu \mathrm{m}$ resolution. The total time of this experiment was about 10 minutes.

\section{Results and Discussion}


The image obtained by the IP is shown in figure 3(a). It is obvious that the radiation field and the laser cross-hairs are superimposed in the same IP directly. Figure 3(b) shows the horizontal profile along the ROI. According to our expectation, PSL signals are decreased at cross-hairs laser positions. The radiation field produced by proton beam irradiation is recorded as "positive" information and the laser position is recorded as "negative" one on an IP. In order to calculate the radiation center, we assumed the intermediate point between both of half height points of dose distribution as the radiation center. It is found that the deviations of radiation center from the intersection of the laser cross-hairs are $0.15 \mathrm{~mm}$ in horizontal and $0.05 \mathrm{~mm}$ in vertical separately. The influence of IP's displacement on the result can be ignored because the relation between the radiation field and the laser position does not depend on the IP's displacement. As a consequence, the easy and short time setup is achieved.

\section{Conclusions}

We have succeeded in the direct measurement for coincidence of the dose distribution and the laser position by using an IP. The IP has advantage such as wide dynamic range, linear response, and high-sensitivity. And the IP has sensitivity for various kinds of beams such as ultraviolet-ray, X-ray, electron, proton, other heavy-ions and neutron. In our method, an easy setup is introduced without an additional object located on the laser position to know the coincidence. The taking time for this measurement is less than 10 minutes. The laser intersection is obviously superimposed as reduced PSL signals in the radiation field on the IP. These are required for daily and monthly quality checks. This technique can be used to align the radiation beam to the laser intersection with excellent accuracy. In addition, this method is available not only for the particular geometric field but also for the actual treatment field. Thus, this method has the potential to become widely available and is useful in standardizing quality assurance method in various external radiation therapy.

\section{References}

1. Tsai J S, Curran B H, Sternick E S and Engler M J 1999 Use of a $1 \mathrm{~mm}$ collimator to test the accuracy of stereostatic radiotherapy Int. J. Radiation Oncol. Biol. Phys. 35 579-86

2. Arjomandy B and Altschuler M D 2000 A quality assurance device for the accuracy of the isocenter of teletherapy and simulation machines Phys. Med. Biol. 45 2207-17

3. Treuer H, Hoevels M, Luyken K, Gierich A, Kocher M, Muller R P and Sturm V 2000 On isocenter adjustment and quality control linear accelerator based radiosurgery with circular collimators and room laser Phys. Med.Biol. $452331-42$ 
4. Welsh K T, Wlodarczyk R A and Reinstein L E 2002 A new geometric and mechanical verification device for LINACs J. Appl.Clin. Med.Phys. 31 54-61

5. Falco T, Lachaine M, Poffendarger B, Podgorsak E B, and Fallone B G 1999 Setup verification in linac-radiosurgery Med.Phys. 26 1972-8

6. Hayakawa H, Amemiya Y, Tada J, Hosono K and Arimoto T 1996 Application of an imaging plate to dose distribution measurement of proton beam Nucl. Instr. and Meth. A 378 627-8

7. Nohtomi A, Terunuma T, Kohno R, Takada Y, Hayakawa Y, Maruhashi A and Sakae T 1999 Response characteristics of an imaging plate to clinical proton beams Nucl. Instr. and Meth. A 424 569-74

8. Nohtomi A, Sakae T, Terunuma T, Kohno R, Takada Y, Hayakawa Y, Maruhashi A and Yasuoka K 2000 Deterioration of imaging plate by proton irradiation and an evidence of its recovery Nucl. Instr. and Meth. A 441 489-93

9. Iwabuchi Y, Mori N, Takahashi K, Matsuda T and Shioyama S 1994 Mechanism of photostimulated luminescence process in BaFBr:Eu2+ phosphors Jpn.J.Appl.Phys. 33 178-85

Figures

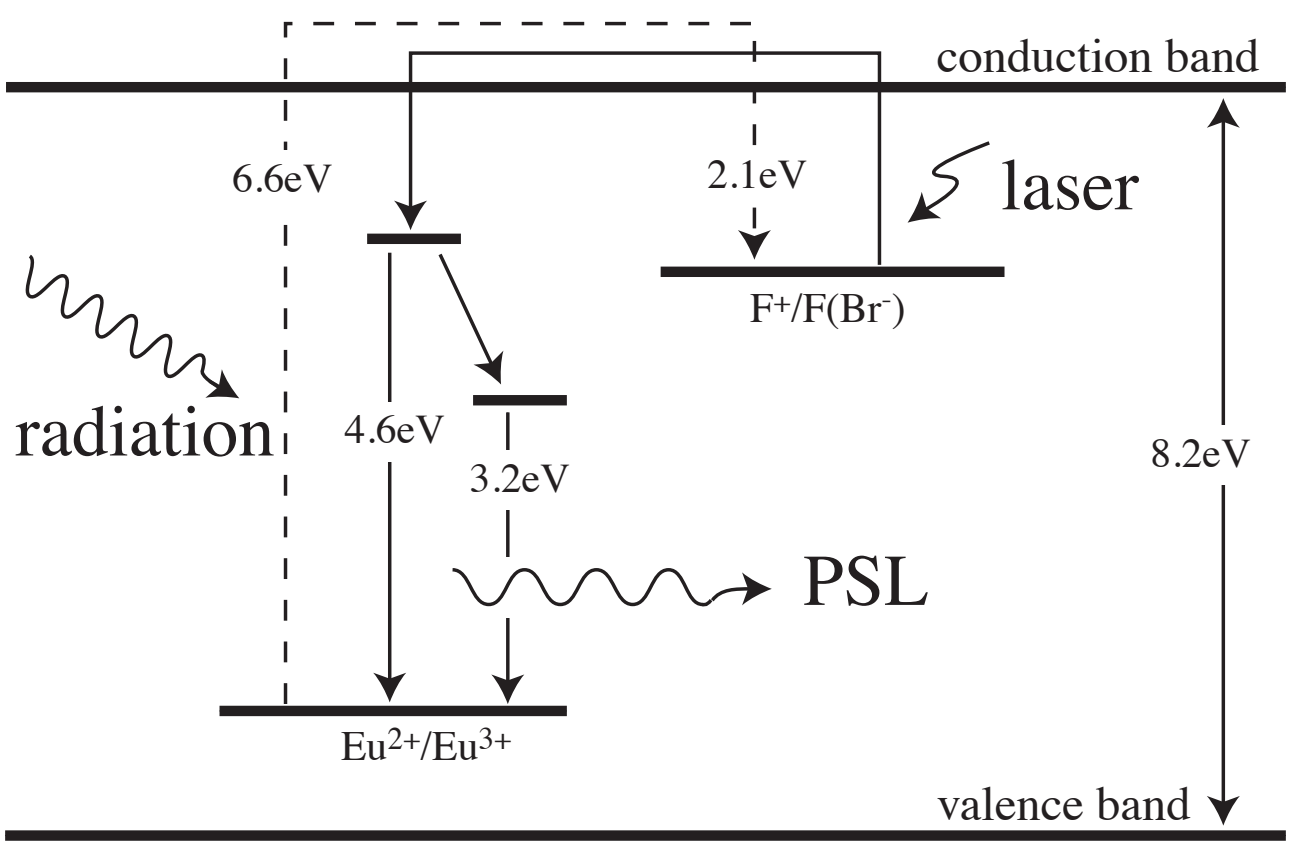

Figure 1. The energy level diagram and the PSL mechanism in BaFBr:Eu ${ }^{2+}$. The dashed line shows ionization and electron trapping process caused by radiation. The solid line shows photoelectric effect and PSL process caused by the laser exposure. 


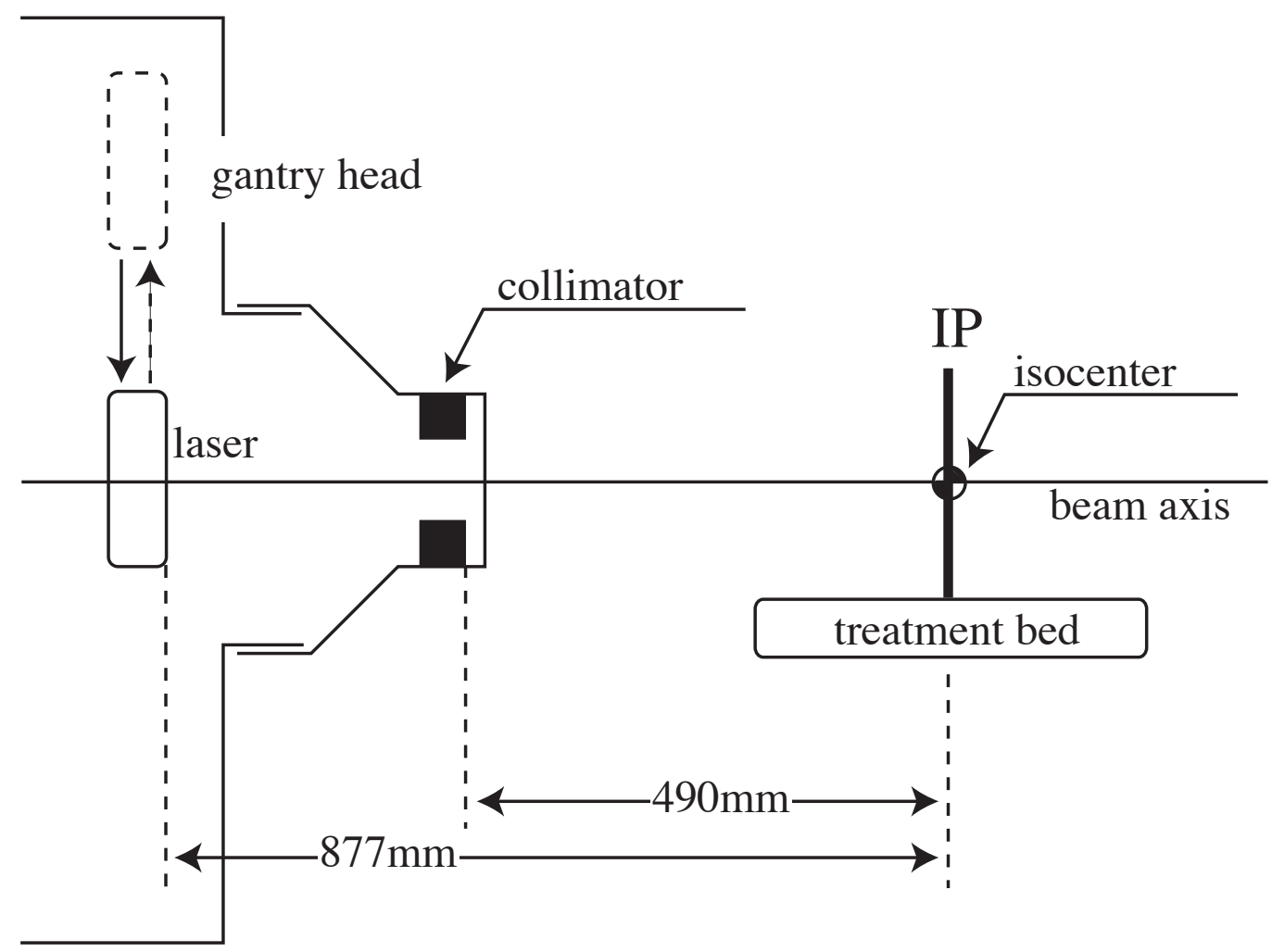

Figure 2. Illustrate of the experiment setup. After proton beam irradiation, the laser system was inserted on the beam line in the gantry head and then the IP was exposed to the cross-hairs laser with wavelength of $635 \mathrm{~nm}$ for about 20 seconds. 

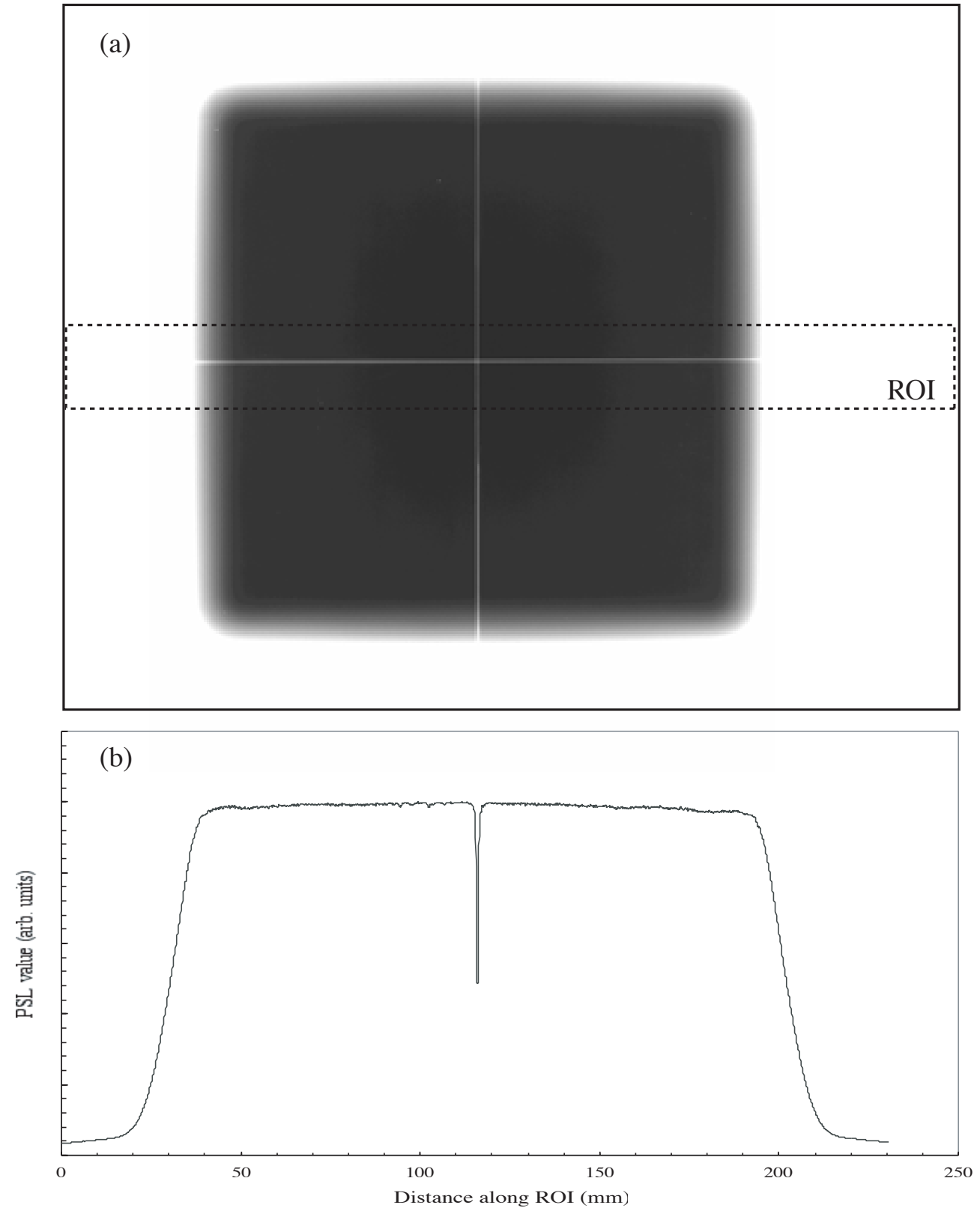

Figure 3. Two-dimensional image of IP (a), and the profile along the horizontal ROI (b). 